\title{
A Model of Glial Scarring Analogous to the Environment of a Traumatically Injured Spinal Cord Using Kainate
}

\author{
Jong Yoon Yoo, MD, $\mathrm{PhD}^{1}$, Chang Ho Hwang, MD, $\mathrm{PhD}^{2}$, Hea Nam Hong, $\mathrm{PhD}^{3}$
}

\author{
${ }^{1}$ Department of Rehabilitation Medicine, Asan Medical Center, University of Ulsan College of Medicine, Seoul; \\ ${ }^{2}$ Department of Physical Medicine and Rehabilitation, Ulsan University Hospital, University of Ulsan College of Medicine, Ulsan; \\ ${ }^{3}$ Department of Anatomy, Asan Medical Center, University of Ulsan College of Medicine, Seoul, Korea
}

\begin{abstract}
Objective To develop an in vitro model analogous to the environment of traumatic spinal cord injury (SCI), the authors evaluated change of astrogliosis following treatments with kainate and/or scratch, and degree of neurite outgrowth after treatment with a kainate inhibitor.

Methods Astrocytes were obtained from the rat spinal cord. Then, 99\% of the cells were confirmed to be GFAPpositive astrocytes. For chemical injury, the cells were treated with kainate at different concentrations (10, 50 or $100 \mu \mathrm{M})$. For mechanical injury, two kinds of uniform scratches were made using a plastic pipette tip by removing strips of cells. For combined injury (S/K), scratch and kainate were provided. Cord neurons from rat embryos were plated onto culture plates immediately after the three kinds of injuries and some cultures were treated with a kainate inhibitor.

Results Astro-gliosis (glial fibrillary acidic protein [GFAP], vimentin, chondroitin sulfate proteoglycan [CSPG], rho-associated protein kinase [ROCK], and ephrin type-A receptor 4 [EphA4]) was most prominent after treatment with $50 \mu \mathrm{M}$ kainate and extensive scratch injury in terms of single arm $(\mathrm{p}<0.001)$ and in the $\mathrm{S} / \mathrm{K}$-induced injury model in view of single or combination $(\mathrm{p}<0.001)$. Neurite outgrowth in the seeded spinal cord ( $\beta$-III tubulin) was the least in the $\mathrm{S} / \mathrm{K}$-induced injury model $(\mathrm{p}<0.001)$ and this inhibition was reversed by the kainate inhibitor $(\mathrm{p}<0.001)$.

Conclusion The current in vitro model combining scratch and kainate induced glial scarring and inhibitory molecules and restricted neurite outgrowth very strongly than either the mechanically or chemically-induced injury model; hence, it may be a useful tool for research on SCI.
\end{abstract}

Keywords Spinal cord injuries, In vitro techniques, Neuroglia, Kainic acid

Received December 15, 2015; Accepted March 14, 2016

Corresponding author: Chang Ho Hwang

Department of Physical Medicine and Rehabilitation, Ulsan University Hospital, University of Ulsan College of Medicine, 877 Bangeojinsunhwan-doro, Dong-gu, Ulsan 44033, Korea. Tel: +82-52-250-7210, Fax: +82-52-250-7211, E-mail: chhwang1220ciba@gmail.com

ORCID: Jong Yoon Yoo (http://orcid.org/0000-0002-5391-6982); Chang Ho Hwang (http://orcid.org/0000-0003-0444-3602); Hea Nam Hong (http:// orcid.org/0000-0003-2421-9050).

() This is an open-access article distributed under the terms of the Creative Commons Attribution Non-Commercial License (http://creativecommons.org/ licenses/by-nc/4.0) which permits unrestricted noncommercial use, distribution, and reproduction in any medium, provided the original work is properly cited. Copyright $\odot 2016$ by Korean Academy of Rehabilitation Medicine 


\section{INTRODUCTION}

The glial scar tissue, which forms at the lesion site, after spinal cord injury (SCI), is composed mainly of 'reactive' astrocytes. Astrogliosis involves marked up-regulation of two intermediate filaments, which are glial fibrillary acidic protein (GFAP) and vimentin [1]. In the reactive state, astrocytes secrete various neuro-inhibitory molecules such as chondroitin sulfate proteoglycans (CSPGs), which are potent inhibitors of axonal re-growth $[1,2]$.

Therefore, many studies, have attempted to examine the mechanism of glial scar formation and reactive astrogliosis, which are the future targets for therapeutic strategies, using an in vitro central nervous system injury model. However, most of the studies have been limited to brain lesions [3-5].

The scratch wound assay has been used to evaluate the wound healing effect or astrocyte motility [6-8], and to assess the induced reactive astrogliosis [4]. Scratched astrocyte culture is thought to have a similar effect as that of the cells on in vivo traumatic injury; in other words, mechanical stress. However, astrogliosis following SCI is associated with not only mechanical injury but also with succeeding neurotoxicity [9]. Following the initial traumatic SCI, excitatory molecules, like glutamate, induce secondary degeneration including reactive astrogliosis and formation of the glial scar [10]. The mechanically disrupted spinal cord is exposed to secondary damage, and this process is promoted by the release of excitatory amino acids (EAAs) such as glutamate [11], which cause excite-toxicity through two classes of ionotropic receptors, the $N$-methyl-D-aspartate (NMDA) receptors and the AMPA ( $\alpha$-amino-3-hydroxy-5-methyl-4-isoxazole propionic acid) / kainate (2-carboxy-4-isopropenyl-pyrrolidin-3-yl acetic acid) receptors. To develop a glial scar model parallel to in vivo glial scar formation, it seems necessary that both mechanical and chemical injuries should be involved. Although kainate (KA) is known to be 30 times more neurotoxic than glutamate [12], it has not been used for developing an in vitro model of astrogliosis except for epilepsy.

The authors aimed (1) to develop an in vitro glial scar model in which both mechanical and chemical injuries were provided and (2) to examine the change in the expression of inhibitory molecules and neurite outgrowth induced by KA treatment in glial scar formation initiated by scratch injury. This is the first trial in terms of usage of KA for developing an in vitro SCI model.

\section{MATERIALS AND METHODS}

Two kinds of experiments were performed. One experiment was performed to determine the optimal type of injury and the other experiment was performed to evaluate neurite outgrowth in spinal cord neurons seeded into astrocytes after different kinds of injury. The laboratory sequences of the former experiment were as follows: initially, astrocytes were obtained from rat pups and cultured, in the second model of chemical injury, KA was applied to the cultured astrocytes at different concentrations $(10,50$ or $100 \mu \mathrm{M})$. In the third model of mechanical injury, two types of scratching times (moderate and extensive) were provided to the other cultured astrocytes. In the fourth model of injury, a combination of chemical (50 $\mu \mathrm{M} \mathrm{KA}$ ) and mechanical (extensive) injuries was applied to the other cultured astrocytes, and finally, immunoblot analyses were performed respectively. The laboratory sequences of the latter experiment were as follows: initially, spinal cord neurons were obtained from embryonic rats, cultured, and seeded into different types of astrocytes injured by KA, scratch, or a combination of the two. In the second experiment, some cultures were treated with a KA inhibitor, and finally immuno-fluorescence analyses were performed to compare the respective degree of neurite outgrowth.

\section{Astrocyte cultures}

All of the following procedures were performed in accordance with the guidelines of the Asan Medical Center Institutional Animal Care Committee protocols of Ulsan University. Astrocyte cultures of spinal cords were obtained from P3-P5 Sprague-Dawley rat pups. Rats were anesthetized using a mixture of xylazine and ketamine (10 mg/kg and $100 \mathrm{mg} / \mathrm{kg}$ ). An incision was performed through the skin and muscles overriding the thoracic spine to expose the T9-T11 vertebrae. The spinal cord (spinal level T11/12) was uncovered through a dorsal laminectomy at T10. The spine was fixed by two Adson forceps either of which was placed at T9 and T11. Meninges and blood vessels were stripped, and the spinal cord was cut into 250- to $400-\mu \mathrm{m}$-thick slices using a McIlwain tissue chopper (Ted Pella Inc., Redding, NY, USA). The 
minced tissues were trypsinized with TrypLE (Invitrogen, Carlsbad, CA, USA) and dissociated by trituration through a fire-polished Pasteur pipette in the presence of $0.02 \%$ DNase (Sigma, St Louis, MO, USA). Cell suspension was filtered through a $80 \mathrm{u}$ cell strainer (BD Falcon; BD, Le Pont de Claix, France) and centrifuged at 1,200 rpm for 1 minute. Cell pellets were seeded on poly-Llysine coated $25 \mathrm{~cm}^{2}$ cell culture flasks (Nunc) or 6-well plates in DMEM (Dulbecco's modified Eagle's medium; Gibco, Carlsbad, CA, USA) supplemented with $20 \mathrm{mM}$ glucose, $15 \%$ fetal bovine serum (FBS, Gibco), and penicillin $(100 \mathrm{IU} / \mathrm{mL}) /$ streptomycin $(100 \mu \mathrm{g} / \mathrm{mL})$ (Invitrogen). After a 1-3 hour adhering interval, loosely adhering and suspended cells (most of which were oligodendroglia) were removed by gently shaking the flasks at room temperature. The strongly adhering microglia were then released by vigorous shaking in $10 \mathrm{mM} \mathrm{L}$-leucine-methyl ester (Sigma), which served as a lysosomotropic agent that destroys mononuclear phagocytic cells. Cells were allowed to grow to near confluence in DMEM containing $20 \mathrm{mM}$ glucose and $10 \%$ calf serum in a water saturated air with $5 \% \mathrm{CO}_{2}$ at $37^{\circ} \mathrm{C}$. When they were nearly confluent, the cultures were passaged by using trypsin, and the media was replaced. To obtain highly differentiated astrocytes, cells were cultured for more than 3 weeks after seeding. After 3 weeks, immunocytochemical staining of representative cultures demonstrated that about $99 \%$ of the cells in these cultures were GFAP-positive astrocytes and approximately $1 \%$ of the cells were CNPase-positive oligodendrocytes, but, OX-42-positive microglia/macrophages were not detected.

\section{Kainate-induced in vitro glial scar model}

Considering that glutamate is known to induce reactive astrogliosis [10] and KA is known to be 30 times more neurotoxic than glutamate [12], authors chose KA for inducing intense astrogliosis. After 3 weeks in vitro, astrocyte cultures were bathed in Krebs solution having the following components: glucose (11 mM), $\mathrm{KCl}(4.5$ $\mathrm{mM}), \mathrm{NaCl}(113 \mathrm{mM}), \mathrm{MgCl}_{2} 7 \mathrm{H}_{2} \mathrm{O}$ (1 mM), $\mathrm{CaCl}_{2}(2 \mathrm{mM})$, $\mathrm{NaH}_{2} \mathrm{PO}_{4}(1 \mathrm{mM}), \mathrm{NaHCO}_{3}(25 \mathrm{mM})$, gassed with $95 \% \mathrm{O}_{2}$ and $5 \% \mathrm{CO}_{2}, \mathrm{pH} 7.4$ at room temperature [13]. KA (Sigma) was dissolved in Krebs solution and cultures were bathed at different concentrations of KA $(10,50$ or $100 \mu \mathrm{M})$ for 2 hours. Sham control cultures were exposed to DMEM only. At the end of the exposure time, cells were rinsed with Hank's balanced salt solution (HBSS; Sigma-Aldrich, Steinheim, Germany) and were maintained in culture for $4,24,48$, and 72 hours before use in experiments with DMEM supplemented with $10 \%$ fetal calf serum (FCS). Six independent experimental sessions using three different concentrations were performed for quantitative analyses.

\section{Scratch wound on astrocytes}

Uniform scratch wounds were made on confluent astrocytes in 6 -well plates using a standard $200 \mu \mathrm{L}$ sterile plastic pipette tip by removing a strip of cells. For the 'moderate' (M) scratch wound model, two horizontal lines and two vertical lines (1 cm apart) were drawn. A grid pattern of three horizontal lines and three vertical lines $(0.8 \mathrm{~cm}$ apart) was made for the 'extensive' (Ex) scratch wound model. Following scratching, the detached cells and debris were rinsed out with HBSS and the medium was replaced with fresh medium. This technique produced a consistent line devoid of cells, approximately $3.3 \mathrm{~cm}$ long $\times 500$ um wide. Four lines were drawn in the 'moderate' wound model, and six lines were produced for the 'extensive' wound model. Approximately $6.9 \%$ and $10.3 \%$ of the total surface areas $\left(9.6 \mathrm{~cm}^{2}\right)$ were denuded in the 'moderate' and the 'extensive' models, respectively. Cultures were incubated for 24,48 , and 72 hours before analysis. Three independent experimental sessions for either of the two injuries were performed for quantitative analyses.

\section{Kainate treatment of scratched astrocytes}

Confluent astrocytes were scratched with a yellow pipette tip $(200 \mu \mathrm{L})$ in a grid pattern of three horizontal lines and three vertical lines resulting in the 'extensive' scratch wound model. To remove cell debris and detached cells, wells were washed with HBSS, followed by 2-hour incubation with $50 \mu \mathrm{M}$ of KA. Cells were rinsed with HBSS and cultured for 24, 48, and 72 hours using DMEM including $10 \%$ FCS. Six independent experiments were performed for quantitative analyses.

\section{Confocal microscopy for the glial scar}

Cells were imaged using a Leica TCS-SP2 laser scanner confocal system (Leica, Heidelberg, Germany) attached to a DMIR2E inverted microscope (Leica Camera AG, Wetzlar, Germany). The FITC label was detected with Ar- 
gon laser at $488 \mathrm{~nm}$, and Cyanine 3 (Cy3) was visualized with red Argon laser at $514 \mathrm{~nm}$. Cells were pretreated with $0.3 \%$ Triton X-100 in 2\% BSA solution for 1 hour. Rabbit anti-GFAP monoclonal antibody (1:1000; Chemicon, Temecula, CA, USA) was applied for 24 hours at $4^{\circ} \mathrm{C}$ and subsequently incubated with anti-rabbit $\mathrm{Cy} 3$-conjugated secondary antibody (1:100; Jackson ImmunoResearch Labs, West Grove, PA, USA) for 2 hours at room temperature. Mouse anti-phosphacan antibody (1:1000, Chemicon) was incubated with anti-mouse FITC-conjugated secondary antibody (1:100, Jackson ImmunoResearch Labs) after application. For negative control, one group of cells was processed without applying primary antibodies. All cells were rinsed several times in phosphate buffer, mounted using Fluoromount (Dako, Carpinteria, CA, USA), and then examined under a Leica confocal laser scanning microscope. Digital images were saved and processed with Adobe Photoshop for final editing.

\section{Immunoblot analysis}

Cells were lysed by adding SDS-PAGE (sodium dodecyl sulfate-polyacrylamide gel electrophoresis) sample buffer (62.5 mM Tris-HCl, pH 6.8, 2\% SDS, 7.8\% glycerol, $4.5 \%$ mercaptoethanol, and $0.1 \%$ bromophenol blue) and were boiled for 5 minutes at $100^{\circ} \mathrm{C}$. Lysates were clarified by centrifugation, and then protein concentrations of the supernatant were determined relative to a bovine serum albumin standard. The cell lysate was subjected to SDS-PAGE on a $4 \%$ stacking gel and a $10 \%$ polyacrylamide separating gel for 70 minutes at $130 \mathrm{~V}$. The protein extracts were transferred onto nitrocellulose membranes with a Bio-Rad transfer unit for 120 minutes at $200 \mathrm{~mA}$. Protein blots were incubated in blocking buffer (2\% BSA in Tween20/TBS) for 1 hour at room temperature on a rotating platform. These blots were sequentially incubated for 24 hours at $4^{\circ} \mathrm{C}$ with primary antibodies to anti-rabbit GFAP (1:1000), rabbit anti-CSPG (1:1000, Chemicon), vimentin (1:200, Rabbit polyclonal IgG; Santa Cruz Biotechnology, Santa Cruz, CA, USA), rho-associated protein kinase (ROCK, 1:100, Rrabbit polyclonal IgG; Santa Cruz Biotechnology), ephrin type-A receptor 4 (EphA4; 1:200, Rabbit polyclonal IgG; Santa Cruz Biotechnology), proinflammatory cytokines-TNF- $\alpha$ (Rabbit polyclonal IgG, Santa Cruz Biotechnology), TGF- $\beta$ (Rabbit polyclonal IgG, Santa Cruz Biotechnology), and Phospho-Smad3 (mothers against decapentaplegic, p-Smad3, Rabbit poly- clonal IgG; Santa Cruz Biotechnology)—and then they were treated with horseradish peroxidase-conjugated secondary antibodies (1:1000, Santa Cruz Biotechnology) or Cy3-conjugated secondary antibody (1:100, Jackson ImmunoResearch Labs) for 1 hour at room temperature, followed by three washes. Immuno-reactive bands were visualized by chemiluminescent reagents (PIERCE, Rockford, IL, USA). Optical density of the bands was analyzed with an Imaging Densitometer (GS-670 model; Bio-Rad, Hercules, CA, USA).

\section{Analysis of neurite outgrowth in the glial scar models}

Neurite outgrowth in KA-, scratch (S)-, and scratch/KA $(\mathrm{S} / \mathrm{K})$-treated astrocytes was compared. The spinal cord neurons were prepared from embryonic day 16 (E16) Sprague-Dawley rats [14]. Briefly, dissected spinal cord tissues were dissociated with $0.25 \%$ trypsin and collected pellets were further dissociated by trituration. The resulting cell mixture was passed through a $40-\mu \mathrm{m}$ nylon mesh and centrifuged for 3 minutes at 1,200 rpm. Cells were seeded at a density of $2 \times 10^{5}$ cells $/ \mathrm{cm}^{2}$ on Nunclon 8-well plates pre-coated with poly-D-lysine and laminin. Cells were maintained in Neurobasal medium with B27 supplement at $37^{\circ} \mathrm{C}$ under $5 \% \mathrm{CO}_{2}$. After 7 days in vitro, spinal neurons were trypsinized and seeded onto the three different types of injured astrocytes to determine whether there was a difference in the amount of neurite outgrowth in the various in vitro glial scar models treated with KA, scratch, and scratch/KA. To investigate the specificity of KA effect, some cultures were treated with a KA inhibitor (6-cyano-nitroquinoxaline-2,3-dione [CNQX], Sigma). Then, cultures were incubated in the Neurobasal medium for 48 hours and fixed with $4 \%$ paraformaldehyde. For analysis of neurite outgrowth, three independent experiments were performed. In each experiment, two wells were used for each set of conditions, and ten fields per well were randomly selected at a magnification of $\times 10$. Neurite outgrowth was evaluated by measuring the area fraction occupied by neurites stained with anti-Tuj 1 (anti-tubulin, $\beta$-III isoform, Millipore) using the ImageJ (ver. 1.43u) image analyzing system (National Institutes of Health, Bethesda, MD, USA). More than three independent experiments were performed for quantitative analysis. 


\section{Statistics}

Adobe Photoshop 7.0 (Adobe Systems, Mountain View, CA) was used to optimize image quality and to prepare figures. More than three independent experiments were performed. The data were analyzed by a one-way analysis of variance (ANOVA) with Tukey-Kramer multiple comparison post-hoc test using GraphPad InStat ver. 3.05 software program (GraphPad, San Diego, CA, USA). Each value was expressed as mean \pm standard deviation. A value of $\mathrm{p}<0.01$ was considered to indicate a statistically significant effect.

\section{RESULTS}

Confirmation of absence of contamination by other cells

Immunocytochemical staining showed that about $99 \%$ of the cells were GFAP-positive astrocytes and approximately $1 \%$ of the cells were CNPase-positive oligodendrocytes, but OX-42-positive microglia/macrophages were not detected (Fig. 1).
Effects of injury intensity on scratch- and kainate-induced astrogliosis

Astrocytic gliosis was significantly increased at each concentration of KA ( $p<0.001$ vs. CON) (Fig. $2 A$ ). The expression of representative markers of astrogliosis (GFAP and vimentin) stained with $\mathrm{KA}$ at $50 \mu \mathrm{M}$ was stronger than that of markers stained with KA at 10 and $100 \mu \mathrm{M}$ at 48 post-treatment hours. The expression of representative inhibitory molecules (CSPG and ROCK) stained with KA at $50 \mu \mathrm{M}$ was stronger than that of molecules stained with $\mathrm{KA}$ at 10 and $100 \mu \mathrm{M}$ at 24 and 48 post-treatment hours, respectively. The representative markers of astrogliosis (GFAP and vimentin) and cytoskeletal inhibitory molecules (EphA4 and ROCK) in the extensive (Ex) scratch injury model were more strongly stained than those in the moderate (M) scratch injury model at 48 and 72 posttreatment hours, and not at 24 hours $(\mathrm{p}<0.001$ vs. $\mathrm{CON})$ (Fig. 2B).

\section{Effects of a single or combined injury on astrogliosis}

Astrogliosis was significantly increased in KA-, S-, and $\mathrm{S} / \mathrm{K}$-induced injury models ( $\mathrm{p}<0.001$ vs. CON). In the $\mathrm{S} /$ $\mathrm{K}$-induced injury model, the representative markers of astrogliosis (GFAP and vimentin) and inhibitory mol-
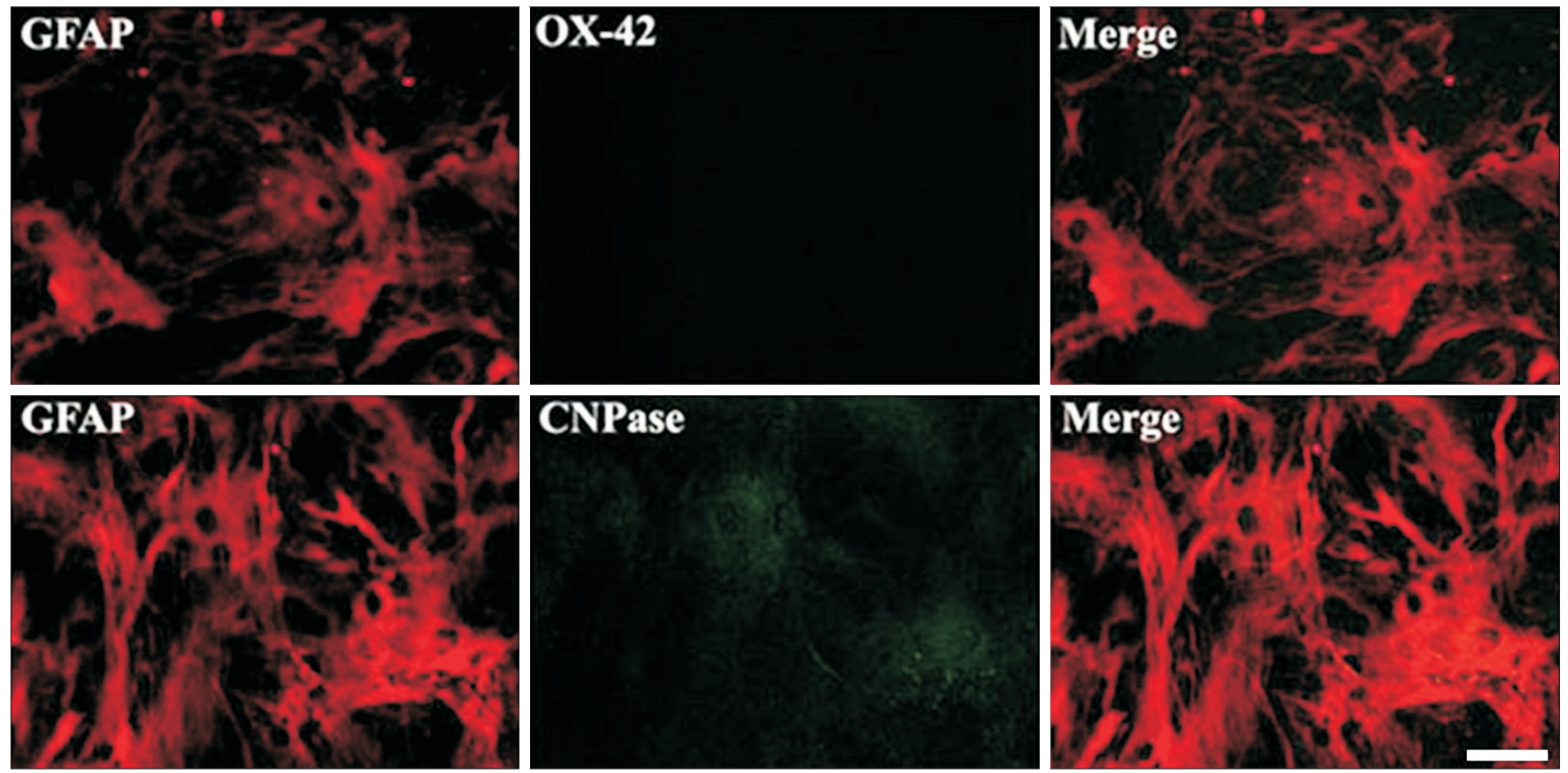

Fig. 1. Confirmation of depletion of microglia. Immunocytochemical staining of the culture system representing a pure astrocyte. GFAP, glial fibrillary acidic protein. 
(A)
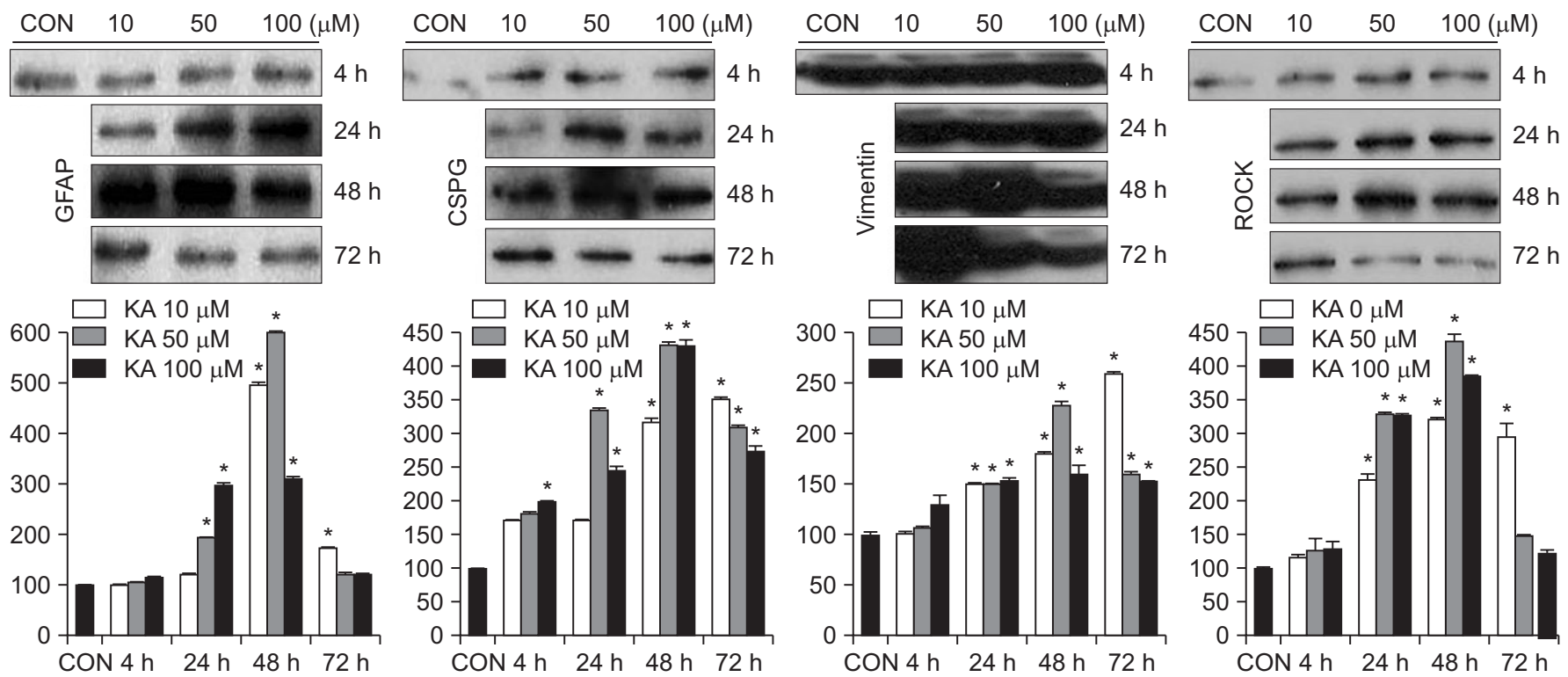

(B)
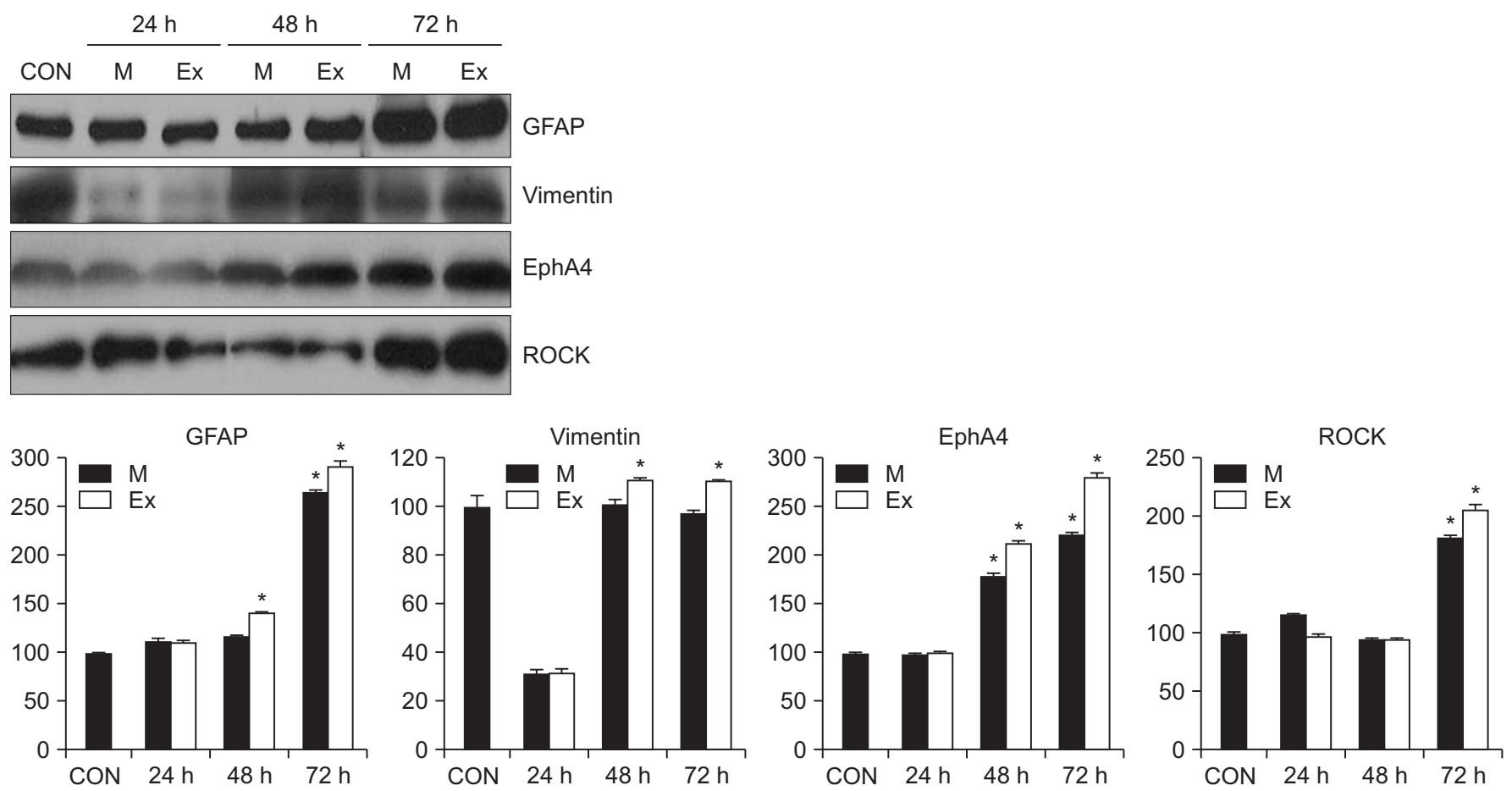

Fig. 2. Effects of injury intensity on scratch- and kainate-induced astrogliosis. (A) The optical immune-densities representing astrogliosis were the strongest at $50 \mu \mathrm{M}$ kainate at 48 post-treatment hours. (B) The optical immune-densities representing astrogliosis were more prominent in the extensive (Ex) scratch injury model than in the moderate (M) scratch injury model. Values are presented as mean \pm standard deviation. GFAP, glial fibrillary acidic protein; CSPG, chondroitin sulfate proteoglycan; ROCK, rho-associated protein kinase; EphA4, ephrin type-A receptor 4; CON, control. ${ }^{*} \mathrm{p}<0.001$ vs. CON; $\mathrm{n}=3-6$. 
ecules (CSPG and ROCK) were more strongly stained and the optical immune-densities were stronger at posttreatment 48 hours than at 24 and 72 hours (Fig. 3).

\section{Effects of kainate on expression of phosphacan}

The area fraction occupied by stained primary antibodies to GFAP and phosphacan representing astrogliosis was the greatest in the S/K-induced injury model among the control, S-, and S/K-induced injury models. The area fraction representative of GFAP and phosphacan in the KA inhibitor (CNQX)-treated S/K-induced injury model was reduced more than that in the $\mathrm{S} / \mathrm{K}$-induced injury model, suggesting that treatment with a KA inhibitor could reverse the scarring effect of KA (Fig. 4).

\section{Effects of the kainate inhibitor on neurite outgrowth}

The neurite length measured in a neurite stained with anti-Tuj1, which represents neurite outgrowth, was smaller in the S/K-induced injury model than in the Sinduced injury model, and the decrement was synergistic rather than additive ( $\mathrm{p}<0.001$ vs. $\mathrm{S}$ ). In the $\mathrm{S} / \mathrm{K}$-induced injury model, the neurite length after CNQX treatment recovered to the value in the S-induced injury model
( $p<0.001$ vs. $S / K$ ), showing that inhibition of KA could re-boost neurite outgrowth (Fig. 5). Highly branched processes of neurites were found in the CNQX-treated S/Kinduced injury model, compared to the simple processes of neurites found in the S/K-induced injury model, and they can be representative of a re-boosting effect.

\section{DISCUSSION}

For realization of an in vitro astrocyte scarring model analogous to the environment of a traumatically injured spinal cord, the authors provided scratch-induced or/ and KA-induced injury to the cultured astrocytes, and checked the pattern of proteoglycan expression according to the intensity and combination of injury mode and neurite outgrowth after seeding into astrocytes. Our key findings are as follows: (1) the optical immune-densities of GFAP, vimentin, CSPG, ROCK and EphA4 are most intense at a $50 \mu \mathrm{M}$ concentration of KA among the 10, 50, and $100 \mu \mathrm{M}$ concentrations, and they are more prominent in the extensive scratch injury model than in the moderate scratch injury model, (2) the optical immunedensities of GFAP, vimentin, CSPG, and ROCK are stron-
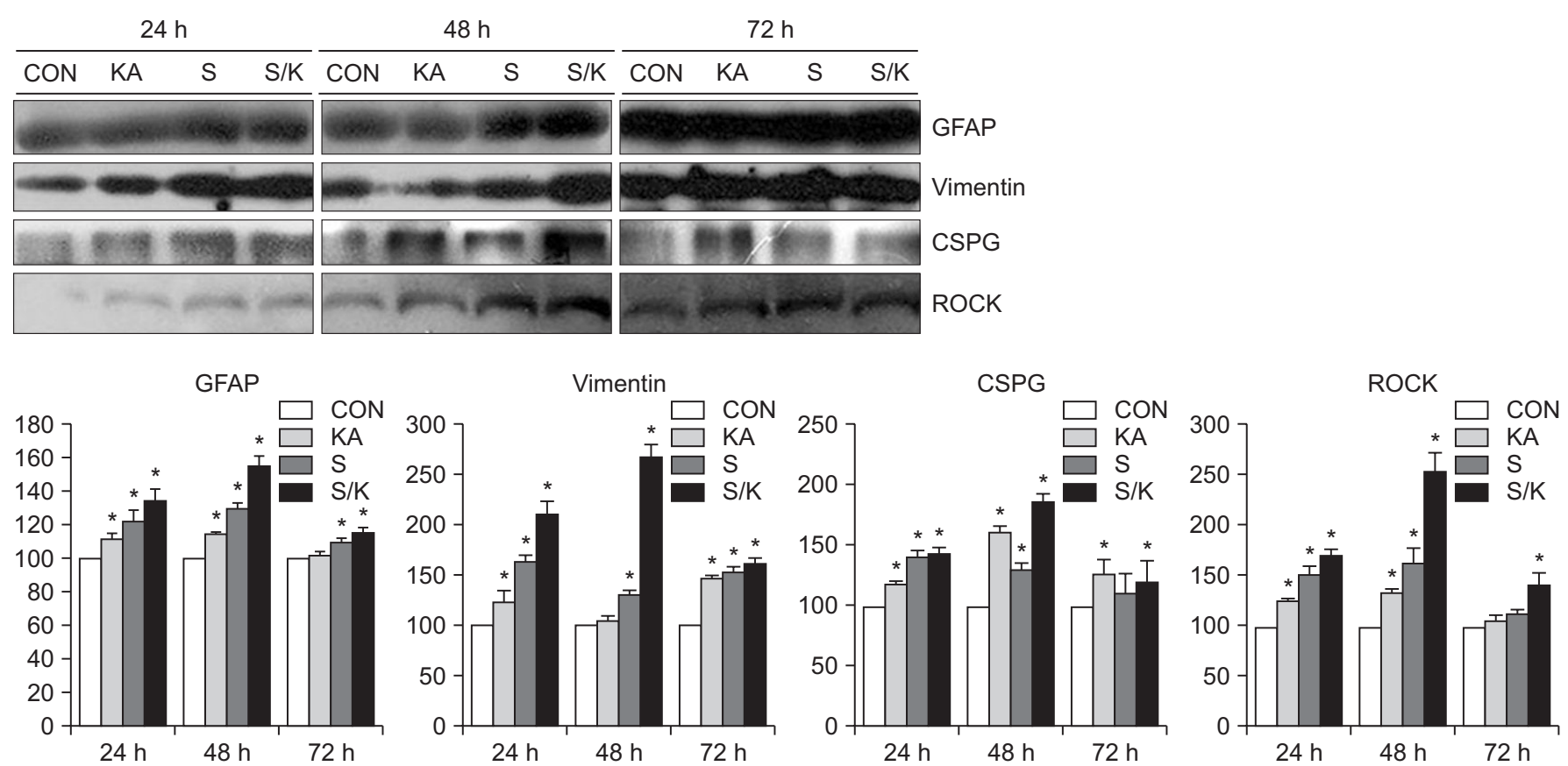

Fig. 3. Effects of single or combined injury on astrogliosis. The optical densities representing glia scarring were the strongest in the combined injury model among the control (CON), chemical (KA), mechanical (S), or combined injury $(\mathrm{S} / \mathrm{K})$ models and glial scarring in the S/K-induced injury model was the most intense at post-treatment 48 hours. Values are presented as mean \pm standard deviation. GFAP, glial fibrillary acidic protein; CSPG, chondroitin sulfate proteoglycan; ROCK, rho-associated protein kinase; KA, kainate; S, scratch; S/K, scratch/kainate. ${ }^{*}$ p $<0.001$ vs. CON; n=3-6. 


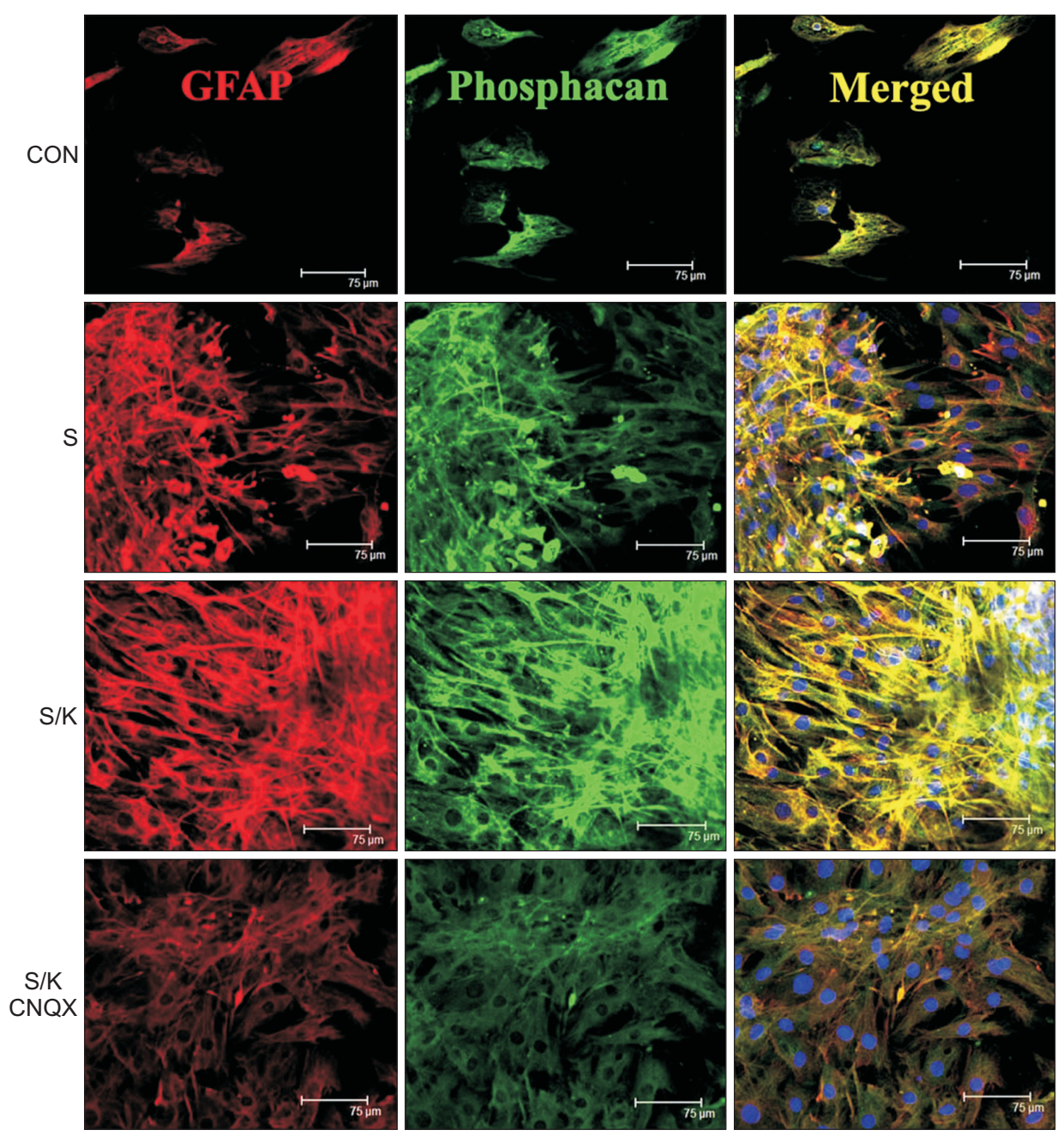

Fig. 4. Effects of kainate on expression of phosphacan (scale bar, 75 $\mu \mathrm{m}$ ). Western blot was performed $(n=3-6$ for immunoblot analysis at each time point in each group). Stained GFAP and phosphacan representing astrogliosis were the largest in the scratch/kainate (S/ $\mathrm{K})$-induced injury model among the control (CON), scratch (S), or S/K-induced injury models. Merged images (Merged) showed double-immunolabeling for GFAP and phosphacan. GFAP, glial fibrillary acidic protein; CNQX, 6-cyano-nitroquinoxaline-2,3dione. ger in the combined injury model than in the singular (scratch or KA) injury model, (3) the immuno-positive area fraction of GFAP and phosphacan is larger in the combined injury model than in the scratch injury model, (4) the immuno-positive area fraction of stained antiTuj1 is smaller in the combined injury model than in the scratch injury model, (5) treatment with a KA inhibitor reverses KA-induced neurite outgrowth inhibition.

Increased proteoglycan expression has been reported in response to mechanical $[7,14]$ and chemical injuries [15]. O'Callaghan et al. [16] reported that the synthesis of GFAP following administration of neuro-toxicants was up-regulated in a dose-dependent manner, and Ellis et al. [17] reported that the degree of injury in rat cortical astrocyte culture exposed to stretch was proportional to the extent of mechanical deformation. These results are compatible with the current results obtained from mechanical injury. As various concentrations of gluta- mate have been reported in physiologic and pathologic conditions $[10,11]$, the authors determined that $50 \mu \mathrm{M}$ is the most ideal concentration for inducing astrogliosis. These 'non-dose-dependent' responses are not compatible with previous reports. However, all of the reports on KA have focused on the brain model of epilepsy, except for the report by Milenkovic et al. [18] in which the additive response was not evaluated. In contrast to previous reports on the variable degree of vulnerability of different populations of brain astrocytes to glutamate, AMPA, and KA [19], in the current study, astrocytes showed an expectable pattern of injury after treatment with KA. These expectable characteristics of the current injury model induced with KA may provide a reliable prototype for physicians to support the SCI experiments.

Astrogliosis was most intense at post-treatment 48 hours and the optical immune-densities of vimentin showed a synergistic effect as well as an additive effect. Malhotra 

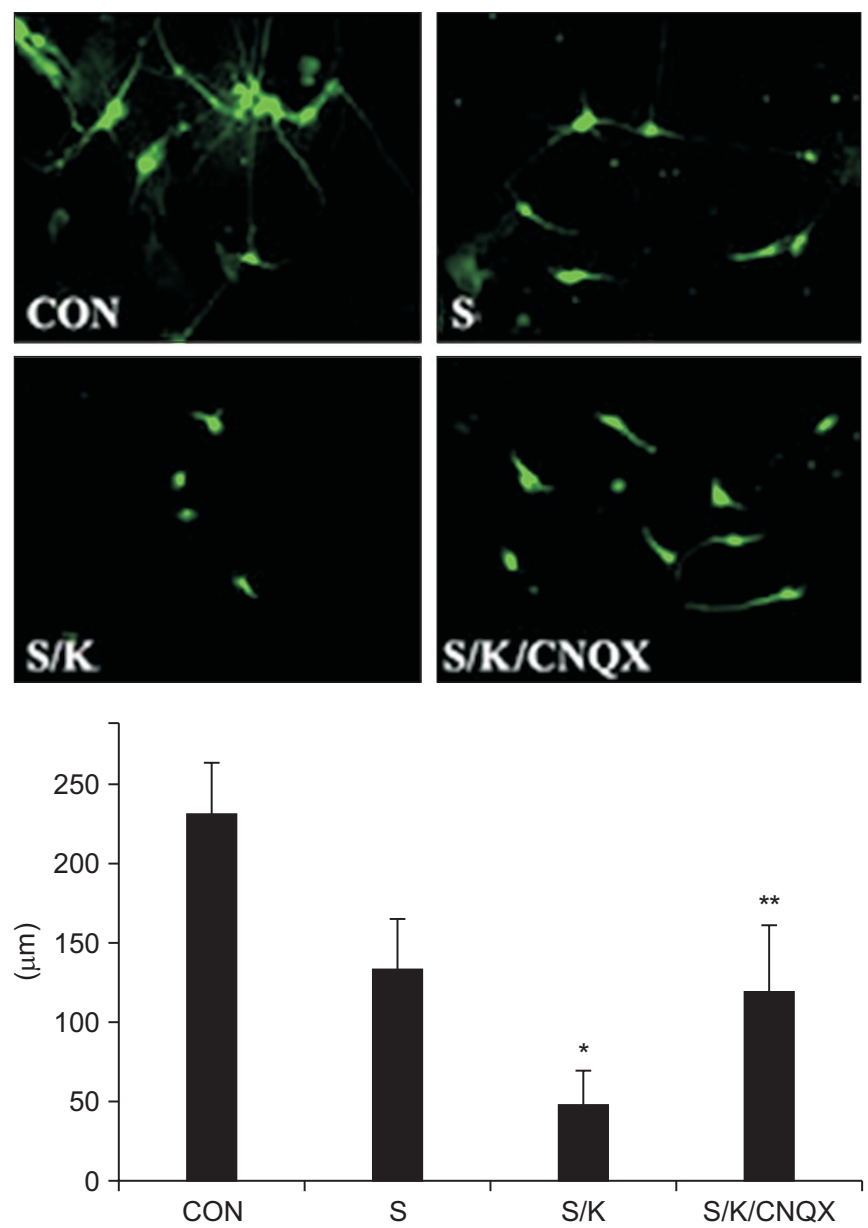

Fig. 5. Effects of the kainate inhibitor on neurite outgrowth. Neurite stained with anti-Tuj1 in the scratch/kainate $(\mathrm{S} / \mathrm{K})$-induced injury model was the smallest among the control (CON), scratch (S), or S/K-induced injury models $\left({ }^{*} \mathrm{p}<0.001\right.$ vs. $\left.S\right)$ and this effect was reversed by treatment with a kainate inhibitor $\left({ }^{* *} \mathrm{p}<0.001 \mathrm{vs.} \mathrm{S} / \mathrm{K}\right)$.

et al. [20] reported that the combination of scratch and CdC12-induced chemical injury induced stronger astrogliosis than only a single injury in cultured 9L rat glioma cells. Similarly, a combination of stretch and co-culture with meningeal fibroblasts produced a widespread glial scar compared to the controlled astrocytes in the study by Wanner et al. [21]. However, although co-culture with fibroblasts displayed the highest mRNA and protein expression of GFAP, phosphacan, neurocan, and tenascin among the controlled astrocytes, stretch and co-culture, their combination did not only cause a significant difference compared to only co-culture but it also showed a paradoxical decrement [21]. It is contrary to the additive effect of combination in the current study. In the current study, in addition to an additive response, a synergistic response of vimentin was observed at post-treatment 48 hours. Additionally, Wanner et al. [21] chose the brain as the target to show that the heterogeneity of response to injury must have occurred. Taking the above comments into consideration, the current combination (scratch and KA) injury model may be superior to the brain injury model invented by Wanner et al. [21] in terms of the degree of astrogliosis and the subsequent evaluation at post-treatment 48 hours is appropriate for an in vitro experiment of astrogliosis.

Wanner et al. [21] reported that combined stretch and co-culture with fibroblasts in post-natal spinal cord neurons resulted in the smallest size of neurite trees per field and it showed a significant difference compared to either of the two. However, it is not compatible with the results of the current study, in which combined injury did not cause a significant difference in the mean length of the longest neurite and the total area of neurite outgrowth per neuron compared to either of the two alone [21]. But, findings of the study by Wanner et al. [21] originated from the experiment in which the brain was used; hence, it could not be compared directly with the current study in spinal cord neurons. Antagonist treatments of the NMDA receptor, one of the EAAs glutamate receptors, limits histological damage and improves functional outcome in the in vivo SCI rat model [22]. More specifically, antagonism of the non-NMDA receptor selectively preferring KA reduces glial loss in the spinal cord contusion model [23] and reduces functional impairment in the in vivo SCI rat model [24]. The above findings indicate the same results as in previous reports which show the potential strength of KA usage in astrogliosis.

Mechanical injury, the disruption of the blood-brain barrier, ischemia, neuro-excitotoxicity, low temperature, laser, and inflammation are contributing factors to scar formation [25-27]. Various in vivo or in vitro culture models have been proposed. in vivo cultures such as insertion of a nitrocellulose sheet into the central nervous system (CNS) have the limitation of lack of easy modulation [28].

Traumatic impact on the CNS causes cellular injury and reactive astrogliosis similar to that caused by mechanical stresses including nerve compression, percussion, and stretch, which have been used for astrocyte activation [21]. Stretch of the tissue, for example, with a rapid pressure pulse using a deformable substrate, induces cul- 
tured cells to transiently increase membrane permeability and to release free radicals [17] and stimulate reactive astrogliosis. Nevertheless, most stretch injury models, except in one report [21], have not proved the expression of neurite outgrowth inhibitors, which is in contrast with the current study. However, the scratch injury model is known to be a well-characterized model for astrogliosis [6-8,14].

Disruption of the blood-brain barrier induces infiltration of macrophages and fibroblasts to form the glial scar [29], and the borders created between astrocytes and meningeal cells shows astrocyte reactivation [30]. Considering that several days are required for the action of co-culture with fibroblasts after the preceding stretch in contrast to KA-induced injury (a few hours), and that there is no significant difference in the average frequency of neurite outgrowth inhibition in the stretch model in the study by Wanner et al. [21], its effect may be more kinetic (mechanical injury-dependent), and therefore it would not be a competitive model for the combination injury model. Glutamate neuro-excitotoxicity is observed in acute CNS injury [31], and it contributes to secondary injury after SCI and it is toxic to astrocytes through glutamate receptor activation [11]. Astrocytes regulate most of the glutamate homeostasis through various glutamate receptors and transporters [10]. These include NMDA, AMPA, and KA receptors as glutamate receptors [10]. KA is known to be 30 times more neurotoxic than glutamate [12]. Glutamate receptors 1-4 subunits compose AMPA receptors, and KA receptors 1-2 subunits and glutamate receptors 5-7 subunits compose KA receptors [10]. Antagonists of AMPA and KA receptors protect neurons more efficiently than those of NMDA receptors [32]. However, AMPA can activate only AMPA receptors, KA can stimulate both AMPA and KA receptors and function independently to AMPA receptor blockers [33]. Because of the degree of strength of KA mentioned above, in the current study, KA treatments in contrast to the no additive effect of the co-culture with meningeal fibroblast model, showed synergistic inhibition of neurite outgrowth such that KA can be intense enough to induce strong astrogliosis, which can be used as an in vitro model.

The glial scar shows permissive as well as inhibitory signals for regenerating axons [34], and astrocytes prevent glutamate neurotoxicity [35]. In response to injury, expressions of KA receptors [36] and GLT-1 glutamate transporters [37] are up-regulated, and the subsequent accumulation of $\mathrm{Ca}^{2+}$ leads to death of activated astrocytes and failure to form a glial scar in the in vivo experiment [38]. Locally in vivo injection of KA did not cause formation of an encapsulating glial scar around the lesion [18] and it showed preserved neuronal orientation [18]. However, evaluation for permissive phenomena was not performed in the current study. Even though there are reports showing that astrocytes can be de-differentiated into stem/precursor cells through an autocrine or paracrine loop in response to injury [14], it needs to be assessed whether these phenomena affect the settlement of astrogliosis. Cultured astrocytes are too immature to simulate astrocyte reactivation and they favor promotion rather than inhibition of neurite outgrowth [39]. In spite of a full incubation period ( $>3$ weeks), the authors did not check the mitotic status of the cultured astrocytes; hence, the possibility of contamination by undifferentiated cells cannot be ruled out completely. Considering the discordance between peak and expression densities of GFAP in the glial scarring model [21], the increased neurite outgrowth in the current study may be the result of cell contraction and cytoskeletal fasciculation, and it may not a real increase [40]. Similar to the report by Wanner et al. [21], neurons show heterogeneous clustering, and hence, the authors' choice of the most neuron-populated spots for evaluation may have underestimated the inhibitory effect. Various neuronal populations react very differently and the same neurons, depending on their size, respond very differently to the scar-like cultures [21]; hence, the current in vitro model does not represent the in vivo whole SCI.

In conclusion, the current in vitro model analogous to the environment of traumatic SCI, using $50 \mu \mathrm{M}$ KA and extensive scratch, may be useful for assessing the mechanism of glial scar formation and for examining variable treatments to augment regeneration after SCI.

\section{CONFLICT OF INTEREST}

No potential conflict of interest relevant to this article was reported.

\section{ACKNOWLEDGMENTS}

This work was supported by a grant (2011-412) from the 
Asan Institute for Life Science, Seoul, Korea.

\section{REFERENCES}

1. Silver J, Miller JH. Regeneration beyond the glial scar. Nat Rev Neurosci 2004;5:146-56.

2. McKeon RJ, Jurynec MJ, Buck CR. The chondroitin sulfate proteoglycans neurocan and phosphacan are expressed by reactive astrocytes in the chronic CNS glial scar. J Neurosci 1999;19:10778-88.

3. Hatten ME, Liem RK, Shelanski ML, Mason CA. Astroglia in CNS injury. Glia 1991;4:233-43.

4. Yu AC, Lee YL, Eng LF. Astrogliosis in culture. I: The model and the effect of antisense oligonucleotides on glial fibrillary acidic protein synthesis. J Neurosci Res 1993;34:295-303.

5. Calvo JL, Carbonell AL, Boya J. Co-expression of glial fibrillary acidic protein and vimentin in reactive astrocytes following brain injury in rats. Brain Res 1991;566:333-6.

6. Fronza M, Heinzmann B, Hamburger M, Laufer S, Merfort I. Determination of the wound healing effect of Calendula extracts using the scratch assay with 3T3 fibroblasts. J Ethnopharmacol 2009;126:463-7.

7. Nishio T, Kawaguchi S, Yamamoto M, Iseda T, Kawasaki $\mathrm{T}$, Hase $\mathrm{T}$. Tenascin- $\mathrm{C}$ regulates proliferation and migration of cultured astrocytes in a scratch wound assay. Neuroscience 2005;132:87-102.

8. Smith AN, Willis E, Chan VT, Muffley LA, Isik FF, Gibran NS, et al. Mesenchymal stem cells induce dermal fibroblast responses to injury. Exp Cell Res 2010;316:48-54.

9. Park E, Velumian AA, Fehlings MG. The role of excitotoxicity in secondary mechanisms of spinal cord injury: a review with an emphasis on the implications for white matter degeneration. J Neurotrauma 2004;21:754-74.

10. Matute C, Domercq M, Sanchez-Gomez MV. Glutamate-mediated glial injury: mechanisms and clinical importance. Glia 2006;53:212-24.

11. Liu D, Xu GY, Pan E, McAdoo DJ. Neurotoxicity of glutamate at the concentration released upon spinal cord injury. Neuroscience 1999;93:1383-9.

12. Olney JW, Rhee V, Ho OL. Kainic acid: a powerful neurotoxic analogue of glutamate. Brain Res 1974;77:50712.
13. Mazzone GL, Margaryan G, Kuzhandaivel A, Nasrabady SE, Mladinic M, Nistri A. Kainate-induced delayed onset of excitotoxicity with functional loss unrelated to the extent of neuronal damage in the in vitro spinal cord. Neuroscience 2010;168:451-62.

14. Yang H, Cheng XP, Li JW, Yao Q, Ju G. De-differentiation response of cultured astrocytes to injury induced by scratch or conditioned culture medium of scratchinsulted astrocytes. Cell Mol Neurobiol 2009;29:45573.

15. Yoshimura E, Majima A, Sakakura Y, Sakakura T, Yoshida T. Expression of tenascin- $\mathrm{C}$ and the integrin alpha 9 subunit in regeneration of rat nasal mucosa after chemical injury: involvement in migration and proliferation of epithelial cells. Histochem Cell Biol 1999;111:259-64.

16. O'Callaghan JP, Jensen KF, Miller DB. Quantitative aspects of drug and toxicant-induced astrogliosis. Neurochem Int 1995;26:115-24.

17. Ellis EF, McKinney JS, Willoughby KA, Liang S, Povlishock JT. A new model for rapid stretch-induced injury of cells in culture: characterization of the model using astrocytes. J Neurotrauma 1995;12:325-39.

18. Milenkovic I, Nedeljkovic N, Filipovic R, Pekovic S, Culic M, Rakic L, et al. Pattern of glial fibrillary acidic protein expression following kainate-induced cerebellar lesion in rats. Neurochem Res 2005;30:207-13.

19. David JC, Yamada KA, Bagwe MR, Goldberg MP. AMPA receptor activation is rapidly toxic to cortical astrocytes when desensitization is blocked. J Neurosci 1996;16:200-9.

20. Malhotra SK, Luong LT, Bhatnagar R, Shnitka TK. Upregulation of reactive astrogliosis in the rat glioma $9 \mathrm{~L}$ cell line by combined mechanical and chemical injuries. Cytobios 1997;89:115-34.

21. Wanner IB, Deik A, Torres M, Rosendahl A, Neary JT, Lemmon VP, et al. A new in vitro model of the glial scar inhibits axon growth. Glia 2008;56:1691-709.

22. Gaviria M, Privat A, d'Arbigny P, Kamenka JM, Haton $\mathrm{H}$, Ohanna F. Neuroprotective effects of gacyclidine after experimental photochemical spinal cord lesion in adult rats: dose-window and time-window effects. J Neurotrauma 2000;17:19-30.

23. Rosenberg LJ, Teng YD, Wrathall JR. 2,3-Dihydroxy6-nitro-7-sulfamoyl-benzo(f)quinoxaline reduces glial loss and acute white matter pathology after experi- 
mental spinal cord contusion. J Neurosci 1999;19:46475.

24. Wrathall JR, Teng YD, Choiniere D. Amelioration of functional deficits from spinal cord trauma with systemically administered NBQX, an antagonist of non$\mathrm{N}$-methyl-D-aspartate receptors. Exp Neurol 1996; 137:119-26.

25. Chang ML, Wu CH, Jiang-Shieh YF, Shieh JY, Wen CY. Reactive changes of retinal astrocytes and Müller glial cells in kainate-induced neuroexcitotoxicity. J Anat 2007;210:54-65.

26. Kohno H, Sakai T, Kitahara K. Induction of nestin, Ki67, and cyclin D1 expression in Müller cells after laser injury in adult rat retina. Graefes Arch Clin Exp Ophthalmol 2006;244:90-5.

27. Schnell L, Fearn S, Klassen H, Schwab ME, Perry VH. Acute inflammatory responses to mechanical lesions in the CNS: differences between brain and spinal cord. Eur J Neurosci 1999;11:3648-58.

28. McKeon RJ, Schreiber RC, Rudge JS, Silver J. Reduction of neurite outgrowth in a model of glial scarring following CNS injury is correlated with the expression of inhibitory molecules on reactive astrocytes. J Neurosci 1991;11:3398-411.

29. Maxwell WL, Follows R, Ashhurst DE, Berry M. The response of the cerebral hemisphere of the rat to injury. II: The neonatal rat. Philos Trans R Soc Lond B Biol Sci 1990;328:501-13.

30. Shearer MC, Niclou SP, Brown D, Asher RA, Holtmaat AJ, Levine JM, et al. The astrocyte/meningeal cell interface is a barrier to neurite outgrowth which can be overcome by manipulation of inhibitory molecules or axonal signalling pathways. Mol Cell Neurosci 2003;24:913-25.

31. Choi DW. Glutamate neurotoxicity and diseases of the nervous system. Neuron 1988;1:623-34.
32. Akins PT, Atkinson RP. Glutamate AMPA receptor antagonist treatment for ischaemic stroke. Curr Med Res Opin 2002;18 Suppl 2:s9-13.

33. Lerma J. Roles and rules of kainate receptors in synaptic transmission. Nat Rev Neurosci 2003;4:481-95.

34. Jones LL, Sajed D, Tuszynski MH. Axonal regeneration through regions of chondroitin sulfate proteoglycan deposition after spinal cord injury: a balance of permissiveness and inhibition. J Neurosci 2003;23:927688.

35. Hansson E, Muyderman H, Leonova J, Allansson L, Sinclair J, Blomstrand F, et al. Astroglia and glutamate in physiology and pathology: aspects on glutamate transport, glutamate-induced cell swelling and gapjunction communication. Neurochem Int 2000;37: 317-29.

36. Gottlieb M, Matute C. Expression of ionotropic glutamate receptor subunits in glial cells of the hippocampal CAl area following transient forebrain ischemia. J Cereb Blood Flow Metab 1997;17:290-300.

37. Simantov R, Crispino M, Hoe W, Broutman G, Tocco G, Rothstein JD, et al. Changes in expression of neuronal and glial glutamate transporters in rat hippocampus following kainate-induced seizure activity. Brain Res Mol Brain Res 1999;65:112-23.

38. Aono S, Kashiwamata S. GFAP under physiological and pathological conditions. Med Sci Res 1990;18:235-9.

39. Smith GM, Rutishauser U, Silver J, Miller RH. Maturation of astrocytes in vitro alters the extent and molecular basis of neurite outgrowth. Dev Biol 1990;138:37790.

40. Faulkner JR, Herrmann JE, Woo MJ, Tansey KE, Doan NB, Sofroniew MV. Reactive astrocytes protect tissue and preserve function after spinal cord injury. J Neurosci 2004;24:2143-55. 